\title{
Intérêt de différents réactifs d'extraction chimique pour l'évaluation de la biodisponibilité des métaux en traces du sol
}

\author{
A Lebourg *, T Sterckeman, H Ciesielski, N Proix \\ Laboratoire d'analyses de sols, Inra, 273, rue de Cambrai, F-62000 Arras, France
}

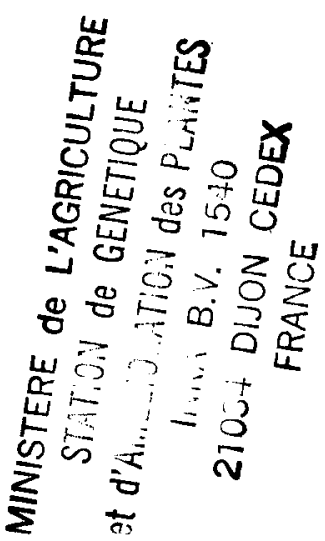

Résumé - L'évaluation de la biodisponibilité des métaux en traces du sol intéresse deux grands domaines d'application : d'une part, le diagnostic de fertilité chimique basé sur l'établissement de seuils de carence, employé depuis plusieurs décennies dans différents pays ; d'autre part, l'estimation du risque de phytotoxicité ou de contamination de la chaîne alimentaire qu'entraîne la pollution du sol par les éléments en traces. Dans ce cas, très peu de pays sont allés jusqu'à l'élaboration de références de diagnostic. Afin de guider le choix d'une méthode d'extraction chimique pour permettre l'ébauche de telles références en France, une synthèse bibliographique a été entreprise. Elle reprend les principaux résultats obtenus depuis les vingt dernières années concernant l'évaluation de la biodisponibilité de $\mathrm{Cd}, \mathrm{Cu}, \mathrm{Zn}, \mathrm{Pb}, \mathrm{Cr}$ et $\mathrm{Ni}$. De cette étude, il ressort que les solutions salines non tamponnées semblent les mieux adaptées à l'estimation rapide du transfert des éléments du sol aux végétaux et à la mise au point de valeurs guides permettant de statuer quant aux risques de toxicité susceptibles d'être engendrés par des sites pollués.

biodisponibilité / sol / métaux en traces / extraction / pollution

Summary - Suitability of chemical extraction to assess risks of toxicity induced by soil trace metal bioavailability. The prediction of soil trace metal bioavailability using extractions has two direct applications: i) evaluation of soil chemical fertility and nutrient deficiency, as has been used widely in different countries for many years; and ii) risk assessment of phytotoxicity and contamination of the food chain induced by polluted soils. In this latter case, few countries have defined guide values. In order to choose one of the extraction methods proposed in the literature, and then define such references for France, a review of research concerning the chemical estimation of Cd, $\mathrm{Cu}, \mathrm{Zn}, \mathrm{Ni}, \mathrm{Cr}$ and $\mathrm{Pb}$ plant uptake in the last twenty years was undertaken. In conclusion, the use of unbuffered salt solutions seems to be the most suitable way to i) estimate trace element transfert from polluted soil to plant and ii) define guide values for risk assessment.

bioavailability / soil / trace metal / extraction / pollution

\footnotetext{
* Correspondance et tirés à part
} 


\section{INTRODUCTION}

Avec le développement de l'activité humaine sont apparus de nouvelles sources et de nouveaux types de pollution. Les contaminants émis par les industries ou apportés avec les épandages agricoles s'accumulent dans le sol et subissent divers processus physiques, chimiques et biologiques, qui peuvent conduire une partie des toxiques à migrer vers les nappes phréatiques ou à s'intégrer à la chaîne alimentaire.

Les éléments en traces tels que, entre autres, le cuivre, le zinc, le chrome et le nickel sont indispensables au développement des végétaux et des animaux. Cependant, à de trop fortes doses, ils peuvent se révéler toxiques. D'autres, tels que le cadmium et le plomb, dont le caractère indispensable n'a pas été démontré, sont potentiellement toxiques (Adriano, 1986 ; Alloway, 1995). Quel que soit l'élément, il importe de préciser dans quelle mesure sa présence constitue ou non un risque pour les différents maillons de la chaîne trophique. Le transfert des éléments du sol aux végétaux peut être estimé par la culture de plantes choisies en fonction de l'activité envisagée sur le site considéré. Les conclusions fournies par ces tests biologiques ont pour avantage d'intégrer l'ensemble des paramètres qui influent sur l'assimilation des métaux en traces (Juste, 1988). Les inconvénients de ces essais sont leur difficulté de mise en œuvre, leur coût relativement élevé et les délais nécessaires à l'obtention de résultats. Afin d'y remédier, de nombreuses méthodes d'extraction chimique relativement simples et rapides ont été mises au point. Leur principe est basé sur un compromis entre la mise en solution plus ou moins sélective des éléments présents dans le sol (solution du sol et fraction échangeable) et la sensibilité analytique des appareils de dosage (Gupta, 1984 ; Juste, 1988). Leur but est de simuler les phénomènes qui contrôlent la biodisponibilité des éléments en traces du sol. Elles nécessitent des normes d'interprétation de l'analyse de sol calées sur la réponse du végétal (Laurent et Castillon, 1989), l'objectif étant de s'affranchir des tests biologiques par la suite. Des relations mathématiques sont donc recherchées entre les quantités de métaux extraites du sol et celles prélevées par les végétaux. Une corrélation significative entre ces données montre que l'extractant est un indicateur valable du risque de transfert des métaux en traces du sol à plante. Cependant, il ne permet pas de mesurer directement la quantité de métal réellement biodisponible.
Les techniques d'extraction ont été initialement développées pour diagnostiquer des carences et sont normalisées dans de nombreux pays (tableau I). Leur application à l'estimation des risques de toxicité est actuellement étudiée (Pays-Bas, Allemagne) ou déjà normalisée (Suisse) (VSBo, 1986 ; Houba et al, 1990 ; Din, 1993). La synthèse bibliographique présentée ici reprend les principaux résultats publiés depuis environ vingt ans concernant les essais d'évaluation de la biodisponibilité de $\mathrm{Cd}, \mathrm{Cr}, \mathrm{Cu}, \mathrm{Ni}, \mathrm{Pb}$ et Zn à l'aide de solutions d'acides dilués, de complexants organiques, d'eau et de solutions salines non tamponnées. L'objectif de ce travail est de contribuer à la définition d'une stratégie de normalisation des méthodes chimiques d'évaluation de la pollution des sols par des métaux potentiellement toxiques.

\section{RAPPEL DE QUELQUES DÉFINITIONS}

Utilisées avec des significations variées, les notions d'extractibilité, de biodisponibilité, de mobilité et de spéciation sont parfois confondues. Aussi, afin d'éviter toute confusion entre ces termes, il nous a semblé nécessaire de les définir.

L'extraction chimique est la mise en solution aqueuse d'une fraction d'un ou de plusieurs éléments présents dans la phase solide du sol. Lorsqu'une seule extraction est pratiquée sur un échantillon de sol, elle est qualifiée "d'extraction simple". En revanche, si plusieurs solutions d'extraction sont utilisées successivement sur un même aliquote de sol, on parle alors "d'extraction séquentielle". Dans chacun de ces cas, l'extraction est suivie du dosage de la totalité de l'élément passé en solution, au moyen d'une technique adaptée (colorimétrie, spectrométrie d'absorption ou d'émission atomique, potentiométrie, etc). Nous avons choisi de ne traiter dans cette synthèse que le cas des extractions simples. Les extractions séquentielles sont difficiles et longues à réaliser. En outre, leur principe de fonctionnement physicochimique est contesté et sujet à controverse. En effet, chaque solution d'extraction est susceptible d'agir sur plusieurs fractions du sol et pas aussi sélectivement que prévu (Hirner, 1992 ; Whalley et Grant, 1993). De plus, lors de la mobilisation d'une forme chimique d'un métal, il se produirait une redistribution de ce métal dans les diverses fractions du sol, ce qui biaiserait les résultats escomptés (Roger, 1986 ; Robbe, 1987 ; Bermond et Malenfant, 
Tableau I. Méthodes d'extraction ménagée des éléments en traces du sol normalisées ou proposées à la normalisation en Europe.

\begin{tabular}{|c|c|c|c|}
\hline Pays & Méthode & Objectif & Référence \\
\hline Allemagne & $\mathrm{NH}_{4} \mathrm{NO}_{3} 1 \mathrm{~mol} . \mathrm{L}^{-1}$ & $\begin{array}{l}\text { Détermination des éléments } \\
\text { en traces mobiles }\end{array}$ & Din, 1993 \\
\hline \multirow[t]{2}{*}{ France } & $\begin{array}{l}\mathrm{Na}_{2} \text {-EDTA } 0,01 \mathrm{~mol}^{-L^{-1}} \\
+\mathrm{CH}_{3} \mathrm{COONH}_{4} 1 \mathrm{~mol}^{-L^{-1}}, \mathrm{pH} 7\end{array}$ & $\begin{array}{l}\text { Évaluation des teneurs assimilables en } \mathrm{Cu} \text {, } \\
\mathrm{Zn} \text { et } \mathrm{Mn} \text { (conseils en fertilisation) }\end{array}$ & Afnor, 1994 \\
\hline & $\begin{array}{l}\text { DTPA 0,005 mol. } \mathrm{L}^{-1}+\text { TEA 0,1 mol. } \mathrm{L}^{-1} \\
+\mathrm{CaCl}_{2} 0,01 \mathrm{~mol} \cdot \mathrm{L}^{-1}, \mathrm{pH}=7,3\end{array}$ & $\begin{array}{l}\text { Évaluation des teneurs assimilables en } \mathrm{Cu} \text {, } \\
\mathrm{Zn}, \mathrm{Fe} \text { et } \mathrm{Mn} \text { (conseils en fertilisation) }\end{array}$ & Afnor, 1994 \\
\hline \multirow[t]{2}{*}{ Italie } & 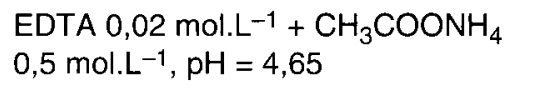 & $\begin{array}{l}\text { Évaluation de la biodisponibilité de } \mathrm{Cu} \\
\mathrm{Zn}, \mathrm{Fe} \text { et } \mathrm{Mn} \text { pour des sols de } \mathrm{pH} \text { acide }\end{array}$ & Unichim, 1991 \\
\hline & $\begin{array}{l}\text { DTPA 0,005 mol.L-1 + TEA 0,1 mol.L } \mathrm{L}^{-1} \\
+\mathrm{CaCl}_{2} 0,01 \mathrm{~mol} \cdot \mathrm{L}^{-1}, \mathrm{pH}=7,3\end{array}$ & $\begin{array}{l}\text { Évaluation de la biodisponibilité } \\
\text { de } \mathrm{Cu}, \mathrm{Zn}, \mathrm{Fe} \text { et } \mathrm{Mn} \text { pour des sols } \\
\text { de } \mathrm{pH} \text { neutre et basique }\end{array}$ & Houba et al, 1990 \\
\hline Pays-Bas & $\mathrm{CaCl}_{2} 0,01 \mathrm{~mol} \cdot \mathrm{L}^{-1}$ & $\begin{array}{l}\text { Évaluation de la biodisponibilité } \\
\text { et de la mobilité des métaux lourds } \\
\text { de sol pollués }\end{array}$ & Houba et al, 1990 \\
\hline Suisse & $\mathrm{NaNO}_{3} 0,1 \mathrm{~mol} \cdot \mathrm{L}^{-1}$ & $\begin{array}{l}\text { Détermination de la teneur soluble } \\
\text { en métaux lourds }(\mathrm{Cu}, \mathrm{Zn}, \mathrm{Cd}, \mathrm{Pb} \text { et } \mathrm{Ni}) \\
\text { et évaluation des risques d'écotoxicité }\end{array}$ & VSBo, 1986 \\
\hline Royaume-Uni & EDTA $0,05 \mathrm{~mol} . \mathrm{L}^{-1} \mathrm{pH}=4$ & Évaluation de la biodisponibilité de $\mathrm{Cu}$ & Maff, 1981 \\
\hline
\end{tabular}

1990 ; Nirel et Morel, 1990 ; Hirner, 1992 ; XiaoQuan et Bin, 1993). Cependant, selon Belzile et al (1989) et Tessier et Campbell (1991), l'erreur ainsi introduite serait négligeable.

On peut définir l'extractibilité comme la faculté d'un élément à passer en solution. Elle est fonction de la solution d'extraction (nature et concentration) et des conditions opératoires suivies (rapport sol/solution, durée et mode d'agitation, température, méthode de centrifugation et de filtration, etc) mais également de la matrice étudiée et de l'état de l'élément dans le sol (Lindsay et Norvell, 1978 ; Sanders et Adams, 1987 ; Alloway et al, 1988 ; Chowdhury et al, 1992 ; He et Singh, 1993 ; Jopony et Young, 1994).

La biodisponibilité est entendue comme la potentialité d'un élément à être absorbé par une plante. Les conditions expérimentales suivies pour l'estimer mettent en œuvre un ensemble de facteurs qui influent fortement sur la capacité des éléments du sol à migrer vers les tissus végétaux. Ainsi, la biodisponibilité est relative à une variété végétale, un stade de développement, des pratiques culturales, au type de sol et à sa profondeur, etc.
La mobilité est définie par la capacité d'un élément à migrer d'un point à un autre, à passer d'une forme chimique à une autre ou à changer de phase (passage de la phase solide à la phase liquide principalement) (Juste, 1988). Cette notion inclut implicitement le facteur temps sans le qualifier de façon précise. Ainsi, Gupta (1992) distingue une fraction mobile (à court terme) et une fraction mobilisable (à long terme).

Enfin, on considère que la spéciation est la caractérisation de la répartition d'un élément dans les différents compartiments du sol ou de l'état chimique dans lequel il se trouve dans ces différents compartiments (ionique, complexé, lié par covalence, etc).

Il est possible de relier entre eux les différents concepts définis. Ainsi, il est admis que la biodisponibilité et la mobilité d'un élément du sol dépendent de sa spéciation (Brümmer et al, 1986). En outre, l'extraction chimique est utilisée pour évaluer la biodisponibilité, la mobilité et la spéciation. En effet, il est classique de comparer les teneurs extraites chimiquement du sol à celles que renferment les végétaux et de rechercher des «corrélations sol-plante». Ces comparaisons peuvent 
être réalisées sur la végétation spontanée ou cultivée. Afin de mieux maîtriser les conditions expérimentales (climatologie en particulier), on préfère généralement les cultures en vase de végétation sous serre aux cultures de plein champ. Cependant, les cultures en pot reproduisent mal les conditions normales d'enracinement et peuvent, en raison du confinement racinaire, perturber l'absorption par le végétal (Jackson et Alloway, 1991). De façon analogue, il peut être envisagé d'estimer la mobilité à partir de l'extractibilité, ce qui nécessite au préalable une étude spécifique du site (caractérisation du bassin versant, étude lysimétrique, simulation de la percolation à l'aide de colonnes de sol...) en vue de la mise en évidence d'une relation extractibilitémobilité valable uniquement dans le cas examiné. Quant à la spéciation, elle est habituellement étudiée au moyen d'extractions séquentielles ou simples. Ainsi, les fractions de métaux considérées comme échangeables, liées à la matière organique, aux carbonates ou à d'autres constituants de la phase solide du sol peuvent être quantifiées, sous réserve des critiques énoncées plus haut. La répartition des éléments dans les différents compartiments du sol et la forme chimique sous laquelle ils se trouvent dans ces compartiments sont également définies à l'aide de modèles ou de techniques analytiques adaptées à la fraction étudiée, passés en revue récemment par Glidewell et Goodman (1995), Greenway (1995), Lumsdon et Evans (1995), Pickering (1995) et Ritchie et Sposito (1995).

\section{PRINCIPALES SOLUTIONS D'EXTRACTION}

Avec l'amélioration des performances des techniques de dosage, les méthodes d'extraction chimique ont évolué vers l'utilisation de solutions de moins en moins agressives vis-à-vis du sol. Ces extractions sont parfois qualifiées de douces ou de ménagées. Dans la littérature publiée lors de ces deux dernières décennies, quatre types de solution d'extraction sont principalement citées : les acides dilués, les complexants organiques, les solutions salines et l'eau pure. Les conditions expérimentales mises en œuvre pour tester la valeur prédictive de ces solutions sont particulièrement variées en ce qui concerne à la fois le protocole d'extraction chimique choisi et les méthodes de culture suivies (espèce végétale, caractéristiques physico-chimiques du sol, culture en serre ou en champ, etc) qui permettent d'établir les corrélations. Or elles influencent fortement les résultats d'estimation de la biodisponi- bilité des éléments en traces du sol. C'est pourquoi elles sont précisées dans la discussion de la plupart des données reprises ici.

\section{Acides dilués}

Les quantités totales de métaux contenus dans le sol sont couramment déterminées en utilisant des acides forts concentrés. En revanche, l'évaluation de la fraction biodisponible est parfois réalisée à l'aide d'acides dilués, comme $\mathrm{CH}_{3} \mathrm{COOH} 0,1 \mathrm{~mol}^{-1}, \mathrm{HCl} 0,1 \mathrm{~mol}^{-1} \mathrm{~L}^{-1}$ ou le mélange de divers acides.

Différentes études citées par Lindsay et Cox (1985), réalisées dans des rizières, montrent une bonne corrélation sol-plante pour $\mathrm{Zn}$ en utilisant $\mathrm{HCl} 0,1$ mol.L $\mathrm{L}^{-1}$. De même, Hughes et Noble (1991) ont observé des relations entre les quantités de $\mathrm{Cr}$ et de $\mathrm{Ni}$ assimilées par des végétaux hyperaccumulateurs se développant sur un sol dérivé de serpentinite et les teneurs extraites du sol par de l'acide acétique $0,1 \mathrm{~mol} . \mathrm{L}^{-1}$. On a également testé un mélange d'acides nommé "Mehlich 1 », composé d'HCl 0,05 mol.L-1 et $\mathrm{d}^{\prime} \mathrm{H}_{2} \mathrm{SO}_{4}$ 0,0125 mol. $\mathrm{L}^{-1}$. Ainsi, dans le cas d'essais sur des sols amendés par des boues, Mulchi et al (1992) ont montré l'existence d'une corrélation entre le contenu en $\mathrm{Zn}, \mathrm{Cu}$, $\mathrm{Ni}$ et $\mathrm{Cd}$ d'extraits de sol et les teneurs relevées dans du tabac. En revanche, la solution "Mehlich 1» ne semble pas adaptée à l'estimation de la biodisponibilité de $\mathrm{Zn}$ vis-à-vis du maîs sur des sols carencés enrichis par du sulfate de zinc (Lins et Cox, 1988). Ceci pourrait s'expliquer par son trop fort pouvoir d'extraction (elle peut extraire les deux tiers du $\mathrm{Zn}$ total) et par le fait que les quantités extraites ne sont pas influencées par les variations de $\mathrm{pH}$ du sol alors que ces dernières agissent sur l'absorption du métal par la plante (Lins et Cox, 1988).

D'une façon générale, l'extraction par les solutions acides diluées se montre trop agressive et peu discriminante vis-à-vis des différentes formes sous lesquelles les éléments en traces sont présents dans le sol. Dans ces conditions, la modélisation de l'absorption de ces éléments par les plantes est rarement réalisable (Juste et Soldâ, 1988 ; Didier et al, 1992).

\section{Complexants organiques}

Les complexants organiques mettraient en solution les éléments échangeables ainsi que ceux complexés principalement par la matière orga- 
nique et ceux fixés sur les hydroxydes du sol (de $\mathrm{Fe}, \mathrm{Mn}, \mathrm{Al}$ ) susceptibles d'être eux-mêmes mis en solution par le réactif (Shuman, 1988 ; Gatti et al, 1991 ; Didier et al, 1992 ; Sheppard et Thibault, 1992). L'EDTA et le DTPA sont les composés les plus utilisés pour évaluer la biodisponibilité de $\mathrm{Cd}, \mathrm{Zn}, \mathrm{Ni}, \mathrm{Cu}$ et $\mathrm{Pb}$. Pour chacun d'eux diverses méthodes sont proposées (Beckett, 1989).

\section{Acide éthylène diaminotétraacétique (EDTA)}

En Angleterre, I'EDTA 0,05 mol. $\mathrm{L}^{-1}$ ajusté à $\mathrm{pH} 4$ a été choisi pour estimer la quantité de $\mathrm{Cu}$ biodisponible (MAFF, 1981). Cette même solution d'extraction semble également satisfaisante pour évaluer la quantité de $\mathrm{Ni}$ assimilable par des végétaux hyperaccumulateurs de métaux se développant sur un sol dérivé de serpentinite (Hughes et Noble, 1991). Quant au BCR (Bureau communautaire des références), il recommande I'EDTA (forme acide) 0,05 mol.L-1 ajusté à $\mathrm{pH} 7$ par addition de soude, pour extraire $\mathrm{Cd}, \mathrm{Cu}, \mathrm{Pb}$, $\mathrm{Ni}$ et $\mathrm{Zn}$. En France, une norme Afnor (Association française de normalisation) propose une solution de $\mathrm{Na}_{2}$-EDTA $0,01 \mathrm{~mol}^{-1}$ dissous dans l'acétate d'ammonium molaire, ajusté à $\mathrm{pH} 7$, afin de définir les teneurs en $\mathrm{Cu}, \mathrm{Zn}$ et $\mathrm{Mn}$ assimilables, en vue de conseils en fertilisation ou de l'évaluation de seuils de carence (Afnor, 1994).

McLaren et al (1984) ont effectivement observé des corrélations sol-plante pour $\mathrm{Cu}$ et pour $\mathrm{Zn}$ extraits par $\mathrm{Na}_{2}$-EDTA 0,04 mol.L-1, ajusté à $\mathrm{pH}$ 6 , lors de l'étude de l'assimilation de ces éléments par de la luzerne présente sur de nombreux sites de Nouvelle-Zélande, potentiellement carencés en $\mathrm{Cu}$ et en $\mathrm{Zn}$. De même, Haq et Miller (1972) ont montré qu'une solution d'EDTA 0,01 mol.L-1 dans $\left(\mathrm{NH}_{4}\right)_{2} \mathrm{CO}_{3} 1 \mathrm{~mol} . \mathrm{L}^{-1}$, ajusté à $\mathrm{pH} 8,6$, permettait d'évaluer la quantité de $\mathrm{Zn}$ susceptible d'être assimilée par le maïs (étude en serre de 85 sols relativement pauvres en zinc).

Mehlich (1984) a mis au point une solution d'extraction, dite "Mehlich 3", renfermant divers composés dont I'EDTA $\left(\mathrm{CH}_{3} \mathrm{COOH} 0,02\right.$ mol.L-1 $+\mathrm{NH}_{4} \mathrm{NO}_{3}$ 0,25 mol.L-1 $+\mathrm{NH}_{4} \mathrm{~F} 0,015 \mathrm{~mol}^{-L^{-1}}+$ $\mathrm{HNO}_{3}$ 0,013 mol.L-1 + EDTA 0,001 mol.L-1). D'après Tucker et Hight (1990), cette méthode a l'avantage de fournir des résultats reproductibles pour l'extraction de $\mathrm{Zn}$ et de $\mathrm{Cu}$. II semble que les teneurs en cuivre extraites d'un sol acide pol- lué soient corrélées avec les teneurs que renferment les racines de Citrus sp (Alva et al, 1993). De même, les essais de Mulchi et al (1992) soulignent l'intérêt d'utiliser cet extractant pour évaluer la biodisponibilité de $\mathrm{Zn}, \mathrm{Cu}, \mathrm{Ni}$ et $\mathrm{Cd}$ de sols pollués, cultivés en tabac.

La méthode de Lakanen et Erviö (1971) (EDTA 0,02 mol.L ${ }^{-1}+\mathrm{CH}_{3} \mathrm{COOH} 0,5 \mathrm{~mol}^{-1}+$ $\mathrm{NH}_{4} \mathrm{CH}_{3} \mathrm{COO} 0,5$ mol.L-1, $\mathrm{pH}$ ajusté à 4,65) se révèle plus ou moins satisfaisante (selon les métaux et les sols considérés) pour estimer la biodisponibilité (Lindsay et Norvell, 1978 ; Lindsay et Cox, 1985 ; Gatti et al, 1991 ; Roca et Pomares, 1991 ; Gupta et Aten, 1993). Son emploi ne serait à prescrire que dans le cas de sols acides, comme cela est souligné dans la norme italienne pour $\mathrm{Fe}, \mathrm{Mn}, \mathrm{Zn}$ et $\mathrm{Cu}$ (Unichim, 1991). Cependant, les quantités ainsi extraites, ou suivant la norme Afnor, se révèlent souvent élevées et peu corrélées aux teneurs réellement biodisponibles (Didier et al, 1992 ; Gupta et Aten, 1993). En effet, ce réactif a tendance à mobiliser les éléments retenus dans des compartiments du sol que les racines ne peuvent atteindre. Une étude de Hogg et al (1993) souligne ainsi que I'utilisation de I'EDTA conduit à surestimer $\mathrm{Cu}$ assimilable. Cette surestimation serait issue de l'affinité entre I'EDTA et le cuivre et de la mise en solution par l'agent chélatant de composés organiques, auxquels le cuivre est fortement lié (Andersson, 1975). Shuman (1988) envisage également que les composés organiques ainsi libérés agissent à leur tour comme des complexants, amplifiant ainsi la mise en solution de $\mathrm{Cu}$. Des résultats comparables obtenus pour $\mathrm{Pb}$ sont expliqués par ces mêmes hypothèses (Andersson, 1975). Pour améliorer les corrélations sol-plante, Sillanpää (1982) propose de corriger la concentration en $\mathrm{Cu}$ extrait par le réactif de Lakanen et Erviö en fonction de la teneur en matière organique du sol. De la même façon, Laurent et Castillon (1989) préconisent d'intégrer aux modèles de prévision de carence en cuivre la teneur en matière organique et/ou la teneur en $\mathrm{P}_{2} \mathrm{O}_{5}$ en fonction du type de sol. En effet, il existe un antagonisme entre le cuivre et la matière organique et, dans une moindre mesure, le phosphore. On note également que la nature même de la matière organique est à considérer : les acides humiques insolubilisent le cuivre, alors que les acides fulviques forment avec cet élément des composés solubles assimilables par les végétaux. 


\section{Acide diéthylène triaminepentaacétique (DTPA)}

Le DTPA possède un pouvoir d'extraction plus faible que celui de l'EDTA mais plus fort que celui des solutions salines (Jackson et Alloway, 1991 ; Roca et Pomares, 1991 ; Gupta et Aten, 1993). Contrairement à ces dernières, le DTPA serait capable de solubiliser une partie des métaux fixés sur les oxydes de manganèse (Shuman, 1988).

Initialement son emploi a été préconisé par Lindsay et Norvell (1978) pour évaluer les seuils de carence en $\mathrm{Fe}, \mathrm{Zn}, \mathrm{Mn}$ et $\mathrm{Cu}$ de sols de $\mathrm{pH}$ neutre à calcaire. Le réactif qu'ils proposent est constitué de DTPA $0,005 \mathrm{~mol}^{\mathrm{L}} \mathrm{L}^{-1}$, de triéthanolamine (TEA) 0,1 mol.L-1 et de $\mathrm{CaCl}_{2}$ 0,01 mol.L-1. La présence de $\mathrm{CaCl}_{2}$ et de TEA dans la solution d'extraction permet d'éviter une dissolution excessive de $\mathrm{CaCO}_{3}$ et la libération trop importante de métaux (O'Connor, 1988). Pour obtenir la complexation optimale de $\mathrm{Fe}, \mathrm{Mn}, \mathrm{Zn}$ et $\mathrm{Cu}$, le $\mathrm{pH}$ de la solution d'extraction est amené à 7,3 ; il correspond également au $\mathrm{pH}$ des sols étudiés (Lindsay et Norvell, 1978). Divers essais de culture (maïs, sorgho et blé) réalisés principalement sous serre, dans des conditions correctes d'utilisation (sols de teneurs faibles en métaux, de $\mathrm{pH}$ neutre à alcalin et application à l'étude de $\mathrm{Fe}$, $\mathrm{Mn}, \mathrm{Zn}$ et $\mathrm{Cu}$ ) ont montré l'intérêt de cette méthode (Haq et Miller, 1972 ; Lindsay et Norvell, 1978 ; Morel et Guckert, 1984 ; Lindsay et Cox, 1985 ; O'Connor, 1988 ; Gatti et al, 1991 ; Roca et Pomares, 1991). Sillanpää (1982) a également mis en évidence de bonnes corrélations sol-plante pour $\mathrm{Cu}$ et pour $\mathrm{Zn}$ lors de l'étude de plus de 3000 sites répartis dans le monde entier, cultivés en blé et en maïs. Ce réactif est utilisé en Italie pour évaluer les teneurs en Fe, Mn, Zn et $\mathrm{Cu}$ assimilables de sols basiques (Unichim, 1991). En France, cette méthode est normalisée pour les mêmes éléments, sans restriction de $\mathrm{pH}$ (Afnor, 1994).

Dans le cas de sols pollués et de métaux autres que $\mathrm{Fe}, \mathrm{Zn}, \mathrm{Mn}$ et $\mathrm{Cu}$, les résultats obtenus avec la méthode d'extraction de Lindsay et Norvell (1978) divergent selon les éléments étudiés et les conditions expérimentales adoptées. Ainsi, Roca et Pomares (1991) ont observé une corrélation sol-plante dans le cas du maïs, de la pomme de terre et de la laitue pour $\mathrm{Cd}, \mathrm{Cu}, \mathrm{Zn}$ et $\mathrm{Ni}$ (mais pas pour $\mathrm{Cr}$ ) sur un sol calcaire pollué par des boues de station d'épuration. Les études de la biodisponibilité de Cd réalisées par Street et al (1977) (culture en serre de maïs sur des sols de $\mathrm{pH}$ neutre à alcalin enrichis en $\mathrm{Cd}$ ) ainsi que par Juste et Tauzin (1992) (culture en serre de ray-grass sur des sols acides amendés par des boues) aboutissent à des conclusions identiques. De même, Adams et Kissel (1989) ont pu relier l'absorption de $\mathrm{Cu}$ et de $\mathrm{Ni}$ (mais pas de $\mathrm{Zn})$ par du soja cultivé sur des sols de $\mathrm{pH}$ acide à neutre ayant reçu des apports de boues et de $\mathrm{Ca}(\mathrm{OH})_{2}$ ou de $\mathrm{FeCl}_{2}$ aux quantités extractibles par le réactif. Brown et al (1989) observent une bonne corrélation entre la concentration en $\mathrm{Ni}$ extrait par le DTPA et celle déterminée dans divers organes de végétaux (grain d'avoine et ray-grass) récoltés après culture en serre sur des sols possédant des $\mathrm{pH}$ différents, enrichis en $\mathrm{Ni}$ et en Cd par l'apport de boue. En revanche, aucune corrélation sol-plante n'est relevée pour le cadmium. D'autres essais ont également révélé l'absence de valeur prédictive du DTPA pour divers sols pollués. Ainsi, Kuo (1990) a constaté une faible corrélation sol-plante entre $\mathrm{Cd}$ extrait par le DTPA et Cd assimilé par des cardes cultivées sur des sols acides amendés par des boues. D'autre part, Hamilton et Westermann (1991) n'observent pas de corrélations entre les concentrations en $\mathrm{Zn}$ extrait et celles que renferment des haricots cultivés sur un sol enrichi en zinc $\left(\mathrm{ZnSO}_{4}\right)$. Enfin, l'assimilation de $\mathrm{Cd}$ et de $\mathrm{Pb}$ par du ray-grass cultivé sous serre sur deux sols acides amendés par une boue ou enrichis par $\mathrm{Pb}\left(\mathrm{NO}_{3}\right)_{2}$ et $\mathrm{Cd}\left(\mathrm{NO}_{3}\right)_{2}$ n'a pu être évaluée à l'aide du DTPA (Hooda et Alloway, 1993). En effet, alors que l'apport de métaux au sol (pollution accidentelle ou enrichissement expérimental) provoque généralement une augmentation de la concentration en ces éléments dans la solution d'extraction, on n'observe pas systématiquement en parallèle un accroissement de l'assimilation par les végétaux (O'Connor, 1988). Pour obtenir une meilleure estimation des teneurs en $\mathrm{Cd}, \mathrm{Zn}$, $\mathrm{Pb}$ et $\mathrm{Cu}$ sur des sols acides, il est recommandé d'introduire les valeurs $\mathrm{du} \mathrm{pH}$, du taux de matière organique et de la teneur en oxydes de fer amorphes dans des modèles simples de prédiction de l'assimilation (Lindsay et Norvell, 1978 ; Morel et Guckert, 1984 ; Kuo, 1990). Néanmoins, il semble que les phénomènes de compétion entre les éléments présents dans les extraits de sols acides ou pollués et la possible saturation de la capacité de complexation du réactif soient un frein à l'estimation des risques de toxicité liés à la biodisponibilité des métaux en traces à l'aide d'un chélatant (Schneider, 1989).

En horticulture, et de même que Lindsay et Norvell, Alt et Peters (1992) suggèrent d'évaluer la biodisponibilité des éléments présents dans le sol à l'aide d'un mélange de $\mathrm{CaCl}_{2} \quad 0,01 \mathrm{~mol}^{\mathrm{L}} \mathrm{L}^{-1}$ 
et de DTPA 0,002 mol.L-1. Ils ont ainsi montré que, pour des sols acides à neutres, enrichis en $\mathrm{Cu}$ et où sont cultivés des chrysanthèmes, la solution d'extraction ne permettait pas d'estimer la teneur en $\mathrm{Cu}$ biodisponible au-delà d'une concentration en cuivre de $0,36 \mathrm{mg} \cdot \mathrm{L}^{-1}$ dans l'extrait de sol. En effet, passé ce seuil, alors que la concentration en $\mathrm{Cu}$ en solution augmente, la teneur dans le végétal demeure relativement constante.

D'après O'Connor (1988), I'utilisation d'une solution de DTPA dans de l'hydrogénocarbonate d'ammonium (AB-DTPA) est restreinte aux mêmes conditions que celles définies dans la méthode de Lindsay et Norvell (sols de teneurs faibles en métaux, de $\mathrm{pH}$ neutre à alcalin et application à l'étude de $\mathrm{Fe}, \mathrm{Mn}, \mathrm{Zn}$ et $\mathrm{Cu}$ ). Cependant, elle aurait l'avantage d'être nettement plus rapide d'exécution ( 15 minutes d'agitation au lieu de 2 heures). De plus, comme les quantités extraites en $\mathrm{Zn}, \mathrm{Pb}, \mathrm{Cd}$ et $\mathrm{Ni}$ par une solution d'AB-DTPA et par le réactif de Lindsay et Norvell sont significativement corrélées, on en a déduit que l'utilisation de AB-DTPA pourrait avantageusement remplacer celle du DTPA (Soltanpour et Workman, 1979 ; Boon et Soltanpour, 1991).

\section{Eau déionisée}

La solution du sol constitue le principal compartiment dans lequel les racines puisent les éléments nutritifs nécessaires au développement de la plante (Coïc et Coppenet, 1989). Ce constat a conduit à suggérer d'utiliser l'eau comme un réactif simulant bien les conditions naturelles.

D'après Didier et al (1992) et Mench et al (1992), l'extraction de $\mathrm{Cd}, \mathrm{Cu}, \mathrm{Zn}, \mathrm{Pb}$ et Ni par de l'eau déionisée permettrait une bonne évaluation des risques de transfert de ces métaux extraits de sols pollués. Cependant cette technique d'extraction pose différents problèmes analytiques. En effet, l'extraction à l'eau ne permet pas une floculation adéquate de la suspension, ce qui perturbe le dosage ou donne des résultats par excès en raison de la présence dans le réactif d'extraction de particules colloïdales en suspension porteuses de l'élément recherché. D'autre part, les mesures sont rendues difficiles du fait des faibles quantités mises en solution et de la présence de phénomènes d'interférence chimique (Sanders et al, 1987 ; Didier et al, 1992). Ce type d'évaluation serait donc plutôt adapté à l'étude affinée de sols pollués et nécessiterait un appareillage de mesure performant.

\section{Solutions salines non tamponnées}

Sous ce terme sont regroupées les solutions de sels divers: $\mathrm{BaCl}_{2}, \mathrm{AlCl}_{3}, \mathrm{CaCl}_{2}, \mathrm{Ca}\left(\mathrm{NO}_{3}\right)_{2}$, $\mathrm{NaNO}_{3}, \mathrm{NH}_{4} \mathrm{NO}_{3}$, etc. Selon les méthodes employées, la concentration en sels des solutions varie généralement de $0,01 \mathrm{~mol}^{-\mathrm{L}^{-1}}$ à $1 \mathrm{~mol} . \mathrm{L}^{-1}$. La solubilisation des métaux se ferait essentiellement par réaction d'échange cationique, le passage en solution pouvant être influencé par les capacités complexantes de l'anion accompagnant ou du cation lui-même.

\section{Chlorures}

L'extraction de $\mathrm{Cd}, \mathrm{Zn}$ et Ni par le $\mathrm{BaCl}_{2} 0,1$ mol. $L^{-1}$ a permis une bonne évaluation de la biodisponibilité de ces éléments vis-à-vis du raygrass dans le cas d'un sol de $\mathrm{pH}$ acide, amendé par des boues de station d'épuration (Juste et Soldâ, 1988). Les teneurs obtenues dans le $\mathrm{BaCl}_{2}$ 0,1 mol.L-1 sont sensiblement les mêmes que celles relevées dans le $\mathrm{CaCl}_{2} 0,1 \mathrm{~mol} \cdot \mathrm{L}^{-1}$.

Dans le but d'extraire le chrome et le nickel de sols dérivés de serpentinite, Hughes et Noble (1991) ont testé $\mathrm{AlCl}_{3}$ 0,3 mol.L-1. Aucune corrélation sol-plante (végétaux en place, hyperaccumulateurs de métaux) n'a été relevée pour le chrome. En revanche, cette solution a permis une estimation correcte de l'assimilation du nickel.

L'efficacité du chlorure de calcium, à différentes concentrations, pour évaluer la biodisponibilité de $\mathrm{Cd}, \mathrm{Zn}$ et $\mathrm{Ni}$, contenus dans des sols pollués et de $\mathrm{pH}$ varié est montrée par diverses études faisant intervenir la culture de multiples espèces végétales (Sauerbeck et Stypereck, 1984 ; Sanders et al, 1987 ; Wu, 1989 ; Mench et al, 1992 ; Mench, 1993 ; Novozamsky et al, 1993). Quelques résultats sont reportés dans le tableau II. Les raisons invoquées pour expliquer cette efficacité, si la solution est centimolaire, sont d'une part la faible modification du pH naturel du sol lors de l'extraction, d'autre part le déplacement aisé par $\mathrm{Ca}^{2+}$ des ions fixés sur le complexe argilo-humique du sol (réaction d'échange) et enfin la similitude entre la composition qualitative et quantitative de la solution du sol avec la solution d'extraction, excepté en ce qui concerne les chlorures (Houba et al, 1990 ; Houba et Novozamsky, 1993). Cependant, d'après Jackson et Alloway (1991), l'extraction de 
Tableau II. Coefficients de détermination de régressions linéaires réalisées entre les concentrations extraites par $\mathrm{CaCl}_{2}$ et les teneurs dans diverses plantes ( ${ }^{*}$ concentrations exprimées en log).

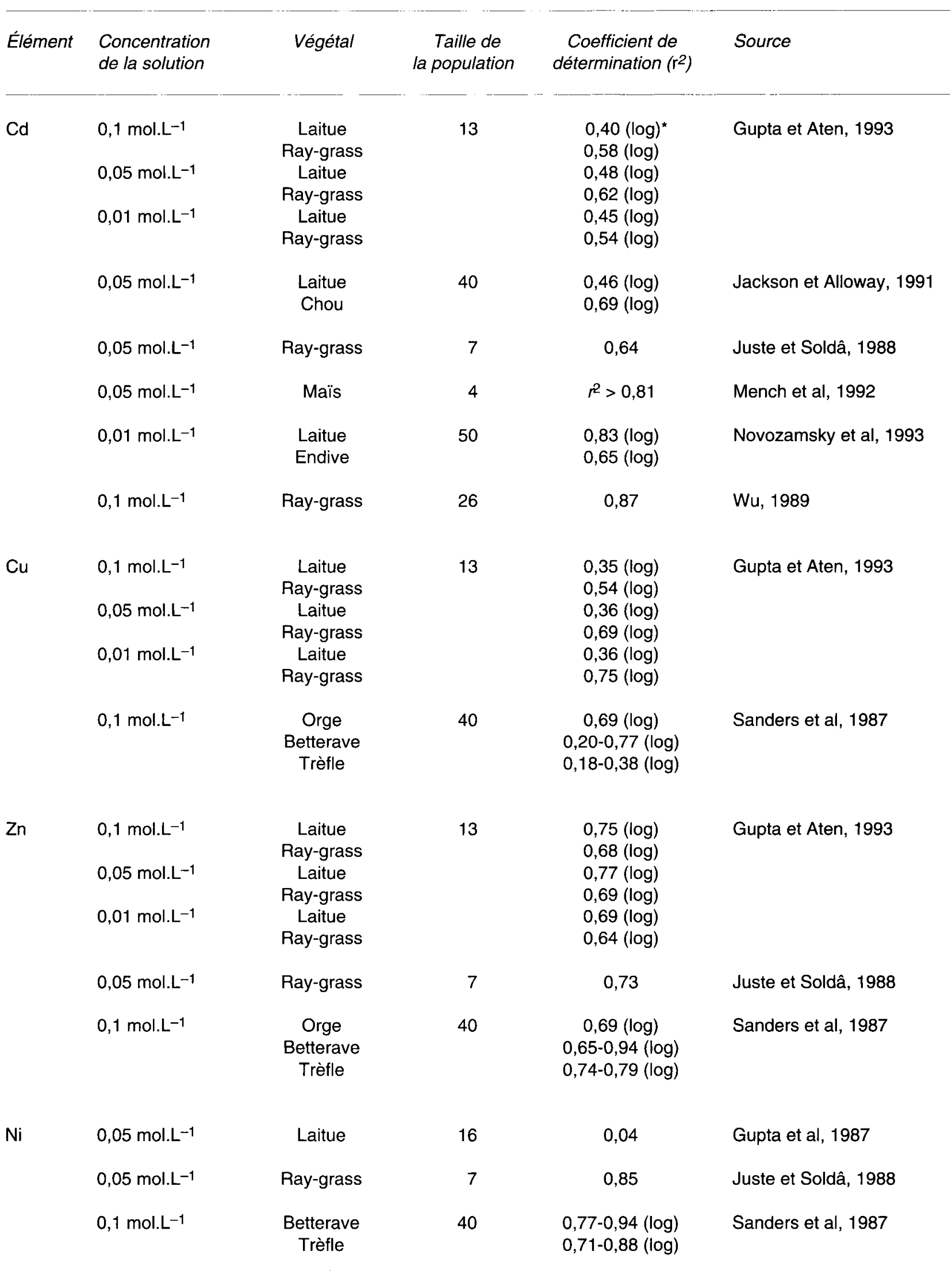


Cd par $\mathrm{CaCl}_{2}$ 0,1 mol.L-1 serait représentative de l'assimilation par les plantes (laitue et chou) seulement si le pH du sol est supérieur à 5,5. De plus, l'emploi de $\mathrm{CaCl}_{2}$ est susceptible de surestimer la fraction extractible de Cd par la formation de complexes avec $\mathrm{Cl}^{-}$(Gupta et Aten, 1993). Pour la même raison, l'évaluation de $\mathrm{Zn}$ par cette méthode est contestée (Gupta et Aten, 1993). Dans le cas de $\mathrm{Cd}$, cet inconvénient peut être limité en intégrant le $\mathrm{pH}$ et la teneur en $\mathrm{Zn}$ (ion en compétition avec $\mathrm{Cd}$ ) dans les équations de corrélation sol-plante (Houba et Novozamsky, 1993). Les teneurs en $\mathrm{Cu}$ extraits par $\mathrm{CaCl}_{2}$ à 0,01 mol. $\mathrm{L}^{-1}$ peuvent se révéler proches des limites de détection et donc difficilement exploitables (Alt et Peters, 1992). Lorsque ce n'est pas le cas, des corrélations significatives entre les concentrations en $\mathrm{Cu}$ dans les extraits de sol et les teneurs dans les végétaux sont parfois observées (tableau II).

\section{Nitrates}

Pour éviter la complexation de l'ion étudié par $\mathrm{Cl}^{-}$ et les problèmes de dosage par spectrométrie d'absorption atomique en four engendrés par la présence de l'ion chlorure (Didier et al, 1992), des solutions de $\mathrm{Ca}\left(\mathrm{NO}_{3}\right)_{2}$, de $\mathrm{NaNO}_{3}$ et de $\mathrm{NH}_{4} \mathrm{NO}_{3}$ sont aussi utilisées. L'emploi de ces solutions a également été développé afin de permettre le dosage des éléments en traces par polarographie, technique pour laquelle l'emploi du nitrate est quasiment indispensable.

Les tests réalisés à l'aide de $\mathrm{Ca}\left(\mathrm{NO}_{3}\right)_{2}$ 0,1 mol. $L^{-1}$ se sont avérés satisfaisants pour $\mathrm{Cd}$, $\mathrm{Zn}, \mathrm{Cu}, \mathrm{Ni}$ et $\mathrm{Pb}$ lors d'essais en serre et au champ, sur des sols acides à neutres pollués (Didier et al, 1992 ; Mench et al, 1992), mais sont encore trop peu nombreux pour permettre d'apprécier l'efficacité de ce réactif.

Le $\mathrm{NaNO}_{3}$ aurait également pour avantage de ne pas perturber le $\mathrm{pH}$ du sol et de ne pas avoir de propriétés complexantes (Gupta et Aten, 1993). Malgré des concentrations en solution parfois proches des limites de détection, Gupta et Aten (1993) montrent que les faibles quantités de $\mathrm{Cd}, \mathrm{Zn}$ et $\mathrm{Cu}$ extraits de sols pollués sont corrélées aux teneurs relevées dans des végétaux (laitue et ray-grass) (tableau III) et sont proches de celles de la solution de sol. De bonnes corrélations ont également été obtenues lors de l'estimation de l'assimilation de Cd par le maïs, les

Tableau III. Coefficients de détermination de régressions linéaires réalisées entre les concentrations extraites par $\mathrm{NaNO}_{3}$ et les teneurs dans diverses plantes ( ${ }^{\star}$ concentrations exprimées en log).

\begin{tabular}{|c|c|c|c|c|c|}
\hline Élément & $\begin{array}{l}\text { Concentration } \\
\text { de la solution }\end{array}$ & Végétal & $\begin{array}{c}\text { Taille de } \\
\text { la population }\end{array}$ & $\begin{array}{c}\text { Coefficient de } \\
\text { détermination }\left(\mathrm{r}^{2}\right)\end{array}$ & Source \\
\hline \multirow[t]{3}{*}{$\mathrm{Cd}$} & $0,1 \mathrm{~mol} . \mathrm{L}^{-1}$ & $\begin{array}{c}\text { Ray-grass } \\
\text { Radis }\end{array}$ & $\begin{array}{l}12 \\
11\end{array}$ & $\begin{array}{l}0,83 \\
0,77\end{array}$ & Gupta, 1984 \\
\hline & $0,1 \mathrm{~mol} . \mathrm{L}^{-1}$ & $\begin{array}{c}\text { Laitue } \\
\text { Ray-grass }\end{array}$ & 43 & $\begin{array}{c}0,48(\log )^{\star} \\
0,44(\log )\end{array}$ & Gupta, 1991 \\
\hline & $0,1 \mathrm{~mol} \cdot \mathrm{L}^{-1}$ & $\begin{array}{c}\text { Laitue } \\
\text { Ray-grass }\end{array}$ & 13 & $\begin{array}{l}0,47(\log ) \\
0,45(\log )\end{array}$ & Gupta et Aten, 1993 \\
\hline \multirow[t]{2}{*}{$\mathrm{Cu}$} & $0,1 \mathrm{~mol} \cdot \mathrm{L}^{-1}$ & $\begin{array}{c}\text { Laitue } \\
\text { Ray-grass }\end{array}$ & 35 & $\begin{array}{l}0,74(\log ) \\
0,66(\log )\end{array}$ & Gupta, 1991 \\
\hline & 0,1 mol.L-1 & $\begin{array}{c}\text { Laitue } \\
\text { Ray-grass }\end{array}$ & 13 & $\begin{array}{l}0,33(\log ) \\
0,78(\log )\end{array}$ & Gupta et Aten, 1993 \\
\hline \multirow[t]{2}{*}{$\mathrm{Zn}$} & $0,1 \mathrm{~mol} . \mathrm{L}^{-1}$ & $\begin{array}{c}\text { Laitue } \\
\text { Ray-grass }\end{array}$ & 35 & $\begin{array}{l}0,71(\log ) \\
0,69(\log )\end{array}$ & Gupta, 1991 \\
\hline & $0,1 \mathrm{~mol} . \mathrm{L}^{-1}$ & $\begin{array}{c}\text { Laitue } \\
\text { Ray-grass }\end{array}$ & 13 & $\begin{array}{l}0,74(\log ) \\
0,57(\log )\end{array}$ & Gupta et Aten, 1993 \\
\hline $\mathrm{Ni}$ & $0,1 \mathrm{~mol} \cdot \mathrm{L}^{-1}$ & Laitue & 15 & 0,31 & Gupta et al, 1987 \\
\hline
\end{tabular}


feuilles et les racines de radis et les feuilles d'épinard (Gupta, 1984 ; Gupta et Stadelmann, 1984), de Cu par le maïs (Gupta, 1987) et de Ni par la laitue (Gupta et al, 1987). Parallèlement, on a observé que l'influence de paramètres tels que le $\mathrm{pH}$ et la capacité d'échange cationique sur les équilibres entre les phases solide et liquide du sol, tout comme les compétitions entre ions ( $\mathrm{Cd}$ et $\mathrm{Pb}$ ) pour les mêmes sites, sont correctement simulées par l'extraction au $\mathrm{NaNO}_{3} 0,1$ mol.L-1 (Gupta et Stadelmann, 1984 ; Gupta, 1987). II en résulte que les concentrations en métaux solubles pouvant induire des concentrations toxiques dans les végétaux sont indépendantes des caractéristiques physicochimiques des sols. Ainsi, après de nombreux essais de culture en serre, des valeurs guides maximales tolérables dans les extraits de sol ont été définies et normalisées en Suisse (VSBo, 1986). Afin de vérifier la pertinence de ces valeurs pour évaluer les risques de toxicité en Cd, Zn et Cu, Gupta (1991) a réalisée une étude sur 25 parcelles polluées où ont été cultivés du ray-grass et de la laitue. II a obtenu des diagnostics satisfaisants, sauf dans le cas de l'assimilation du cadmium par la laitue.
En effet, il a relevé des teneurs élevées dans les feuilles alors que les quantités de $\mathrm{Cd}$ extrait des sols sont très faibles. Concernant le cuivre, la valeur seuil définie s'avère trop forte pour distinguer correctement, sur l'ensemble des sols, tout risque de toxicité. Le cas du plomb n'est que rarement abordé. En effet, les concentrations assimilées par les végétaux sont parfois trop faibles pour définir toute relation sol-plante (Gupta et Aten, 1993).

Une étude de Symeonides et McRae (1977) souligne l'efficacité de $\mathrm{NH}_{4} \mathrm{NO}_{3} 1 \mathrm{~mol} . \mathrm{L}^{-1}$ pour évaluer la biodisponibilité de $\mathrm{Cd}$ pour des radis cultivés sur des sols enrichis par l'ajout de $\mathrm{CdCl}_{2}$ (tableau IV). Le travail de Prüess (1992) montre que l'extraction de nombreux éléments en traces du sol par cette même solution est corrélée significativement aux teneurs en ces éléments dans divers végétaux. À partir de ces recherches, un projet de normalisation de la méthode d'extraction par $\mathrm{NH}_{4} \mathrm{NO}_{3}$ a été proposé sous la référence DIN V 19730 (DIN, 1993). Néanmoins, lors des essais de Jackson et Alloway (1991) et de Singh et al (1995), cette méthode s'est révélée peu appropriée à l'évaluation de l'assimilation de Cd

Tableau IV. Coefficients de détermination de régressions linéaires réalisées entre les concentrations extraites par $\mathrm{NH}_{4} \mathrm{NO}_{3}$ et les teneurs dans diverses plantes ( ${ }^{\star}$ concentrations exprimées en log; ns : corrélation non significative).

\begin{tabular}{|c|c|c|c|c|c|}
\hline Élément & $\begin{array}{l}\text { Concentration } \\
\text { de la solution }\end{array}$ & Végétal & $\begin{array}{c}\text { Taille de } \\
\text { la population }\end{array}$ & $\begin{array}{c}\text { Coefficient de } \\
\text { détermination }\left(\mathrm{r}^{2}\right)\end{array}$ & Source \\
\hline \multirow[t]{4}{*}{$\mathrm{Cd}$} & $\begin{array}{l}0,5 \mathrm{~mol} \cdot \mathrm{L}^{-1} \\
0,1 \mathrm{~mol} \cdot \mathrm{L}^{-1}\end{array}$ & $\begin{array}{l}\text { Laitue } \\
\text { Ray-grass } \\
\text { Laitue } \\
\text { Ray-grass }\end{array}$ & 13 & $\begin{array}{c}0,37(\log )^{\star} \\
0,49(\log ) \\
0,38(\log ) \\
0,48(\log )\end{array}$ & Gupta et Aten, 1993 \\
\hline & $1 \mathrm{~mol}^{-\mathrm{L}^{-1}}$ & $\begin{array}{l}\text { Laitue } \\
\text { Chou }\end{array}$ & 40 & $\begin{array}{l}0,22(\log ) \\
0,52(\log )\end{array}$ & Jackson et Alloway, 1991 \\
\hline & $1 \mathrm{~mol} \cdot \mathrm{L}^{-1}$ & $\begin{array}{l}\text { Blé } \\
\text { Carotte } \\
\text { Laitue }\end{array}$ & 12 & $\begin{array}{c}\text { ns- } 0,33 \\
0,23-0,53 \\
0,23-n s\end{array}$ & Singh et al, 1995 \\
\hline & $1 \mathrm{~mol} \cdot \mathrm{L}^{-1}$ & Radis & 75 & 0,94 & Symeonides et McRae, 1977 \\
\hline $\mathrm{Cu}$ & $\begin{array}{l}0,5 \mathrm{~mol} \cdot \mathrm{L}^{-1} \\
0,1 \mathrm{~mol} \cdot \mathrm{L}^{-1}\end{array}$ & $\begin{array}{l}\text { Laitue } \\
\text { Ray-grass } \\
\text { Laitue } \\
\text { Ray-grass }\end{array}$ & 13 & $\begin{array}{l}0,27(\log ) \\
0,64(\log ) \\
0,30(\log ) \\
0,71(\log )\end{array}$ & Gupta et Aten, 1993 \\
\hline $\mathrm{Zn}$ & $\begin{array}{l}0,5 \mathrm{~mol} \cdot \mathrm{L}^{-1} \\
0,1 \mathrm{~mol} \cdot \mathrm{L}^{-1}\end{array}$ & $\begin{array}{l}\text { Laitue } \\
\text { Ray-grass } \\
\text { Laitue } \\
\text { Ray-grass }\end{array}$ & 13 & $\begin{array}{l}0,81(\log ) \\
0,62(\log ) \\
0,73(\log ) \\
0,47(\log )\end{array}$ & Gupta et Aten, 1993 \\
\hline
\end{tabular}


par la laitue, le chou, le blé et la carotte cultivés sous serre sur des sols de $\mathrm{pH}$ varié amendés ou non par des boues. Enfin, l'utilisation de $\mathrm{NH}_{4} \mathrm{NO}_{3}$ 0,1 mol. $\mathrm{L}^{-1}$ et $0,5 \mathrm{~mol} . \mathrm{L}^{-1}$ pour évaluer la biodisponibilité de $\mathrm{Cd}$, $\mathrm{Cu}$ et $\mathrm{Zn}$ vis-à-vis du ray-grass et de laitue cultivés sur différents sites pollués ne semble pas systématiquement adaptée (Gupta et Aten, 1993). Ces échecs auraient principalement deux causes : la solution a tendance à modifier le $\mathrm{pH}$ du sol et l'ammoniac est susceptible de complexer $\mathrm{Cu}, \mathrm{Ni}$ et $\mathrm{Cr}$ (Gupta et Aten, 1993) ainsi que $\mathrm{Cd}$ et $\mathrm{Zn}$.

\section{DISCUSSION}

II apparaît intéressant de comparer les méthodes d'extraction entre elles et plus particulièrement celles employant $\mathrm{CaCl}_{2}, \mathrm{NaNO}_{3}$ et $\mathrm{NH}_{4} \mathrm{NO}_{3}$, solutions qui sont les plus fréquemment utilisées.

D'un point de vue analytique, les quantités extraites doivent être suffisantes afin de réduire les problèmes de mesure liés à des concentrations proches ou en dessous des limites de détection. Ainsi, du fait des faibles quantités extraites, il apparaît délicat d'appliquer ces méthodes d'extraction aux sols dont les teneurs en éléments en traces sont de l'ordre de grandeur du fond géochimique (Sauerbeck et Stypereck, 1984). La comparaison de différentes solutions d'extraction, toutes à la concentration de 0,1 mol.L-1, met en évidence un pouvoir d'extraction croissant selon l'ordre moyen suivant : $\mathrm{Ca}\left(\mathrm{NO}_{3}\right)_{2}<\mathrm{BaCl}_{2}=\mathrm{CaCl}_{2}$ (Juste et Soldâ, 1988 ; Mench et al, 1992). Gupta et Aten (1993) ont observé que $\mathrm{CaCl}_{2} 0,1$ mol. $\mathrm{L}^{-1}$ extrait d'avantage de cuivre, de cadmium, de zinc et de plomb que $\mathrm{NH}_{4} \mathrm{NO}_{3}$ et $\mathrm{NaNO}_{3}$ à la même molarité. Novozamsky et al (1993) ont également montré que pour $\mathrm{Cd}$, $\mathrm{Cu}$ et $\mathrm{Zn}$ la capacité d'extraction de $\mathrm{CaCl}_{2}$ 0,01 mol. $\mathrm{L}^{-1}$ est plus forte que celle de $\mathrm{NaNO}_{3}$ 0,1 mol.L $\mathrm{L}^{-1}$. Il convient cependant de préciser que dans ce cas les ratios sol/solution, les temps d'agitation et les étapes de centrifugation et de filtration diffèrent selon les méthodes. Par conséquent, cette comparaison ne permet pas de juger rigoureusement la capacité d'extraction d'une solution mais plutôt le protocole analytique dans son ensemble. Cependant, d'une manière générale, l'extractibilité des éléments en traces du sol semble plus élevée avec $\mathrm{CaCl}_{2}$ qu'avec $\mathrm{NH}_{4} \mathrm{NO}_{3}$ et $\mathrm{NaNO}_{3}$, si l'on considère des solutions de concentration maximale de 0,1 mol.L-1.

D'autre part, afin de doser les faibles teneurs en solution dans les extraits de sol, la spectrométrie d'absorption atomique électrothermique est généralement employée. On constate alors que les dosages en milieux $\mathrm{NaNO}_{3}$ et $\mathrm{NH}_{4} \mathrm{NO}_{3}$ sont particulièrement sujets à des interférences chimiques et provoquent une détérioration rapide des fours (Gupta et Aten, 1993 ; Houba et Novozamsky, 1993). Le $\mathrm{CaCl}_{2}$ semble aussi poser quelques problèmes analytiques, surtout s'il est utilisé à une concentration décimolaire, du fait de la présence de chlorure (Didier et al, 1992 ; Houba et Novozamsky, 1993). Enfin, par un exercice d'intercalibration Houba (1995) a mis en évidence la faible reproductibilité de ces méthodes d'extraction, ce qui souligne la nécessité d'améliorer les différentes étapes du protocole d'extraction et la qualité des dosages.

Dans les tableaux II, III et IV sont rassemblés les résultats des essais de culture ayant permis de comparer les quantités de métaux polluants extraits par des solutions de $\mathrm{CaCl}_{2}, \mathrm{NH}_{4} \mathrm{NO}_{3}$ et celles assimilées par différents végétaux. Les valeurs extrêmes des coefficients de détermination des régressions linéaires définies entre ces données (exprimées en log des concentrations) pour $\mathrm{Cd}, \mathrm{Cu}, \mathrm{Zn}$ et $\mathrm{Ni}$ sont reportées dans le tableau V. Seuls les résultats obtenus pour le zinc sont relativement homogènes : la fourchette des coefficients de détermination des régressions linéaires est sensiblement la même quel que soit le milieu d'extraction. L'étude de Cu four-

Tableau V. Étendue des coefficients de détermination de régressions linéaires réalisées entre les concentrations extraites du sol ( $\mathrm{log})$ et les teneurs dans divers végétaux $(\mathrm{log})$, définie à partir des données des tableaux II, III et IV.

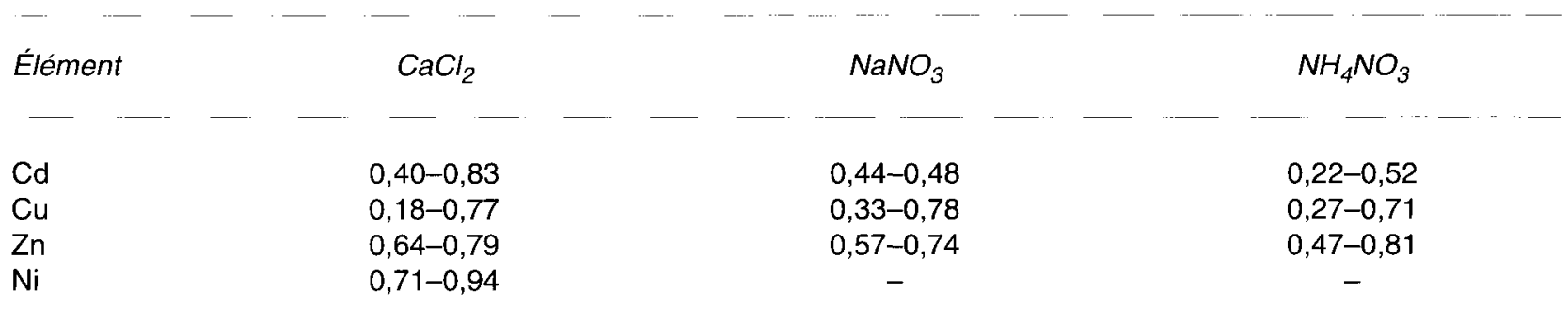


nit la gamme de coefficients la plus large de toutes dans chacune des solutions, ce qui souligne la forte influence des conditions expérimentales adoptées sur les résultats. Quant au cadmium, les meilleures corrélations sol-plante déterminées semblent celles définies en milieu $\mathrm{CaCl}_{2}$. Cependant, aucune des solutions considérées ne s'avère mieux adaptée qu'une autre à l'évaluation de la biodisponibilité de $\mathrm{Cd}, \mathrm{Cu}, \mathrm{Zn}$ et $\mathrm{Ni}$ si l'on se réfère à l'ensemble des données rapportées.

\section{CONCLUSION}

Le choix d'une méthode d'extraction, dans l'objectif de diagnostiquer les risques de toxicité liés à la biodisponibilité des métaux en traces de sols pollués, peut être basé sur deux critères : d'une part l'étendue du domaine d'application de la méthode (nombre de métaux en traces, nature des sols et des végétaux) et d'autre part les limites analytiques de son emploi, point qui est rarement traité dans la littérature.

L'utilisation d'acides dilués pour évaluer la biodisponibilité des métaux en traces n'est généralement pas satisfaisante et serait uniquement adaptée à l'étude de certains sols acides.

Les solutions d'extraction renfermant de I'EDTA, dont l'emploi est normalisé dans différents pays dans le but de formuler des diagnostics agronomiques, se montrent rarement adaptées à l'évaluation des teneurs en métaux biodisponibles de sites pollués. En effet, ce complexant a tendance à surestimer les teneurs en éléments assimilables (surtout le cuivre et le plomb) et permet rarement de définir une corrélation sol-plante. De même, l'utilisation de réactifs contenant du DTPA ne s'applique qu'à des sols de $\mathrm{pH}$ basique à neutre et pour l'étude de carences en $\mathrm{Cu}$ et en $\mathrm{Zn}$ (ainsi qu'en Fe et en Mn). L'emploi de chélatant pour l'étude des sols acides et d'un plus grand nombre d'éléments serait envisageable en faisant intervenir d'autres paramètres du sol (notamment le $\mathrm{pH}$ et la teneur en matière organique) dans les modèles reliant la concentration dans l'extrait de sol à l'assimilation des métaux en traces par les végétaux. Cette application nécessite également la mise au point d'un protocole d'extraction permettant de s'affranchir de la saturation de la capacité complexante du réactif et des phénomènes de compétition entre éléments présents dans les extraits de sol.

L'extraction des métaux polluants du sol par de l'eau déionisée ne paraît pas adaptée à l'éva- luation de la biodisponibilité de ces éléments du fait des diverses difficultés analytiques qu'elle induit.

Les effets d'une contamination en $\mathrm{Pb}, \mathrm{Cr}$ et $\mathrm{Ni}$ sont rarement évalués à l'aide de solutions salines non tamponnées. Par ailleurs, la variabilité des conditions expérimentales suivies pour estimer la biodisponibilité et les contradictions relevées dans les résultats ne permettent pas de définir clairement le domaine d'application des trois réactifs les plus employés $\left(\mathrm{CaCl}_{2}, \mathrm{NaNO}_{3}\right.$ et $\mathrm{NH}_{4} \mathrm{NO}_{3}$ ). Cependant, il semble que l'évaluation de la biodisponibilité des métaux en traces du sol à l'aide de ces méthodes soit exclusivement réservée à l'étude de sols pollués. Dans ce cas, on montre souvent pour $\mathrm{Cd}$, Cu et $\mathrm{Zn}$ des corrélations significatives entre les concentrations dans l'extrait de sol et les teneurs renfermées par des végétaux cultivés sur ce sol. Par conséquent, il serait possible de déterminer, pour certains éléments, une correspondance entre la concentration maximale acceptable dans un tissu végétal défini et la quantité d'élément extrait du sol par une solution saline non tamponnée. Des valeurs guides ont ainsi été élaborées en Suisse à partir des teneurs extraites par $\mathrm{NaNO}_{3} \quad 0,1$ mol.L-1 (VSBo, 1986). Les principales critiques formulées vis-à-vis de l'emploi de solutions salines concernent les différents problèmes analytiques qu'elles occasionnent.

En conclusion, il n'existe pas de méthodes d'extraction chimique universelle permettant d'évaluer simultanément et sans difficultés analytiques l'absorption de $\mathrm{Cd}, \mathrm{Cu}, \mathrm{Zn}, \mathrm{Cr}$, Ni et $\mathrm{Pb}$ par tout végétal cultivé sur des sols pollués et de définir ainsi les risques de toxicité liés à la biodisponibilité de ces éléments. Cependant, l'élaboration d'une norme n'est pas totalement à rejeter. Elle nécessite non seulement d'opter pour une méthode d'extraction, en fonction des avantages et inconvénients de chacune, mais aussi de déterminer le référentiel d'interprétation correspondant à l'aide d'un test biologique. II se pose alors le problème du choix de la ou des plantes cultivées, des organes étudiés, des conditions de culture et de la méthode d'interprétation des données obtenues.

\section{REMERCIEMENTS}

Le travail présenté a pu être réalisé grâce au soutien financier de l'Inra et de la région Nord-Pas-de-Calais. 


\section{RÉFÉRENCES}

Adams JF, Kissel DE (1989) Zinc, copper and nickel availabilities as determined by soil solution and DTPA extraction of a sludge-amended soil. Commun Soil Sci Plant Anal 20, 139-158

Adriano DC (1986) Trace Elements in the Terrestrial Environment. Springer-Verlag, New York

Afnor (Association française de normalisation) (1994) Normes NFX 31-120 et NFX 31-121, Recueil de normes françaises, Qualité des sols, Afnor, Paris, 83-96

Alloway BJ (1995) Heavy Metals in Soils, 2nd ed. Blackie Academic and Professional, Glasgow

Alloway BJ, Thornton I, Smart GA, Sherlock JC, Quinn MJ (1988) Metal availability. The Science of the Total Environment 75, 41-69

Alt D, Peters I (1992) Die $\mathrm{CaCl}_{2}$ /DTPA-Methode zur Untersuchung gärtnerischer Erden auf Mengenund Spurenelemente. Agribiol Res 45, 215-224

Alva AK, Graham JH, Tucker DPH (1993) Role of calcium in amelioration of copper phytotoxicity for Citrus. Soil Sci 155, 211-218

Andersson A (1975) Relative efficiency of nine different soil extractants. Swedish J Agric Res 5, 125135

Beckett PHT (1989) The use of extractants in studies on trace metals in soils, sewage sludges and sludge-treated soils. Adv Soil Sci 9, 143-176

Belzile N, Lecomte P, Tessier A (1989) Testing readsorption of trace elements during partial chemical extractions of bottom sediments. Environ Sci Technol 23, 1015-1020

Bermond A, Malenfant C (1990) Estimation des cations métalliques liés à la matière organique à l'aide de réactifs chimiques: approche cinétique. Sci Sol 28, 43-51

Boon DY, Soltanpour PN (1991) Estimating total $\mathrm{Pb}$, $\mathrm{Cd}$ and $\mathrm{Zn}$ in contaminated soils from $\mathrm{NH}_{4} \mathrm{HCO}_{3}-$ DTPA-extractable levels. Commun Soil Sci Plant Anal 22, 369-378

Brown PH, Dunemann L, Schulz R, Marschner H (1989) Influence of redox potential and plant species on the uptake of nickel and cadmium from soils. Z Pflanzenernähr Bodenk 152, 85-91

Brümmer GW, Gerth J, Herms U (1986) Heavy metal species, mobility and availability in soils. $Z$ Pflanzenermähr Bodenk 149, 382-398

Chowdhury AK, McLaren RG, Swift RS, Cameron KC (1992) The amount of zinc extracted from soils by different extractants. Commun Soil Sci Plant Anal 23, 1451-1459

Coïc $Y$, Coppenet $M$ (1989) Les oligoéléments en agriculture et élevage. Incidences sur la nutrition humaine. Inra, Paris

Didier V, Gomez A, Mench M, Masson P, Lambrot C (1992) Contrôle de la mobilité et de la biodisponibilité des métaux de sols contaminés: efficacité de différents composés du fer et d'autres composés minéraux. Convention de recherche 90-231 Inra/ministère de l'Environnement

DIN (Deutsches Institut für Normung) (1993) Bodenbeschaffenheit. Ammoniumnitratextraktion zur Bestimmung mobiler Spurenelemente in Mineralböden. Vornorm DIN V 19 730. In : Boden Chemische Bodenuntersuchungsverfahren, DIN, Berlin

Gatti M, Anguissola Scotti I, Silva S (1991) Evaluation of soil extractants to estimate available micronutrients under wheat-field conditions. Commun Soil Sci Plant Anal 22, 1883-1893

Glidewell SM, Goodman BA (1995) Direct methods of metal speciation. In : Chemical Speciation in the Environment (AM Ure, CM Davidson, eds), Blackie Academic and Professional, Glasgow, 33-64

Greenway GM (1995) Hybrid methods of speciation. In : Chemical Speciation in the Environment (AM Ure, CM Davidson, eds), Blackie Academic and Professional, Glasgow, 65-85

Gupta SK (1984) Importance of soil composition in deciding the best suitable analytical criteria for guidelines on maximum tolerable metal load and in assessing bio-significance of metals in soils. Schweiz Landw For 23, 3, 209-225

Gupta SK (1987) Interrelationship between degree of metal binding capacity of sludge and metal concentration in plants. In : Heavy Metals in the Environment 1, Int Conf, New Orleans (SE Lindberg, TC Huntchinson, eds), 418-420

Gupta SK (1991) Assessment of exotoxicological risk of accumulated metals in soils with the help of chemical methods standardized through biological tests. In : Trace Metals in the Environment 1 (JP Vernet, ed), Elsevier Publisher, Amsterdam, 55-65

Gupta SK (1992) Mobilizable metal in anthropogenic contaminated soils and its ecological significance. In : Trace Metals in the Environment 2 (JP Vernet, ed), Elsevier-Publisher, Amsterdam, 299-310

Gupta SK, Aten C (1993) Comparison and evaluation of extraction media and their suitability in a simple model to predict the biological relevance of heavy metal concentrations in contaminated soils. Intern $J$ Environ Anal Chem 51, 25-46

Gupta SK, Stadelmann FX (1984) Effect of sewage sludge on the biorelevant cadmium concentration. In : Processing and use of sewage sludge, Proceedings of the 3rd International Symposium, Brighton (P L'Hermite, H Ott, eds), Commission of the European Communities, Bruxelles, 435-445

Gupta SK, Häni H, Stantschi E, Stadelmann FX (1987) The effect of graded doses of nickel on the yield, the nickel content of lettuce and the soil respiration. Toxicol Environ Chem 14, 1-10

Hamilton MA, Westermann DT (1991) Comparison of DTPA and resin extractable soil $\mathrm{Zn}$ to plant $\mathrm{Zn}$ uptake. Commun Soil Sci Plant Anal 22, 5 et 6, 517-528

Haq AU, Miller NH (1972) Prediction of available soil $\mathrm{Zn}, \mathrm{Cu}$ and $\mathrm{Mn}$ using chemical extractants. Agron $\mathrm{J}$ $64,779-782$ 
He QB, Singh BR (1993) Effect of organic matter on the distribution, extractibility and uptake of cadmium in soils. J Soil Sci 44, 641-650

Hirner AV (1992) Trace element speciation in soils and sediments using sequential chemical extraction methods. Intern J Environ Anal Chem 46, 77-85

Hogg D, Mc Laren RG, Swift RS (1993) Desorption of copper from some New Zealand soils. Soil Sci Soc $A m$ J 57, 361-366

Hooda PS, Alloway BJ (1993) Effects of time and temperature on the bioavailability of $\mathrm{Cd}$ and $\mathrm{Pb}$ from sludge-amended soils. J Soil Sci 44, 1, 97-110

Houba VJG (1995) Mobile trace elements. Discussion paper for ISO/TC 190/SC 3/1 N131

Houba VJG, Novozamsky I (1993) State of the art and future developments of soil analysis for bioavailability purposes. Discussion paper for ISO/TC 190/SC 3/1 N76

Houba VJG, Novozamsky I, Lexmond TM, Van Der Lee JJ (1990) Applicability of $0.01 \mathrm{M} \mathrm{CaCl}_{2}$ as a single extraction solution for the assessment of the nutrient status of soils and other diagnostic purposes. Commun Soil Sci Plant Anal 21, 2281-2290

Hughes JC, Noble AD (1991) Extraction of chronium, nickel and iron and the availability of chronium and nickel to plants from the eastern transvaal as revealed by various single and sequential extraction techniques. Commun Soil Sci Plant Anal 22, 1753-1766

Jackson AP, Alloway BJ (1991) The bioavailability of cadmium to lettuce and cabbage in soils previously treated with sewage sludges. Plant Soil 132, 179186

Jopony M, Young SD (1994) The solid-solution equilibria of lead and cadmium in polluted soils. Eur $J$ Soil Sci 45, 59-70

Juste C (1988) Appréciation de la mobilité et de la biodisponibilité des éléments en traces du sol. Science Sol 26, 103-112

Juste C, Soldâ P (1988) Influence de l'addition de différentes matières fertilisantes sur la biodisponibilité du cadmium, du manganèse, du nickel et du zinc contenus dans un sol sableux amendé par des boues de station d'épuration. agronomie 8, 897-904

Juste C, Tauzin J (1992) Comparaison de la biodisponibilité du cadmium contenu dans différentes matières fertilisantes. CR Acad Agric Fr 3, 71-79

Kuo S (1990) Cadmium buffering capacity and accumulation in Swiss chard in some sludge-amended soils. Soil Sci Soc Am J 54, 86-91

Lakanen E, Erviö R (1971) A comparison of eight extractants for the determination of plant available micronutrients in soils. Acta Agr Fenn 123, 223-232

Laurent F, Castillon P (1989) Diagnostic des situations à risque de carence en cuivre pour les céréales. In : Les oligoéléments et le sol (P Duc, ed), Frontières, Paris, 97-107

Lindsay WL, Norwell WA (1978) Development of a DTPA soil test for zinc, iron, manganese, and copper. Soil Sci Soc Am J 42, 421-428
Lindsay WL, Cox FR (1985) Micronutrient soil testing for the tropics. Fertilizer Research 7, 169-200

Lins IDG, Cox FR (1988) Effect of soil pH and clay content on the zinc soil test interpretation for corn. Soil Sci Soc Am J 52, 1681-1685

Lumsdon DG, Evans LJ (1995) Predicting chemical speciation and computer simulation. In : Chemical Speciation in the Environment (AM Ure, CM Davidson, eds), Blackie Academic and Professional, Glasgow, 86-134

MAFF (Ministry of Agriculture, Fisheries and Food) (1981) The Analyses of Agricultural Materials. Reference Book 427, MAFF, Londres

McLaren RG, Swift RS, Quin BF (1984) EDTA-extractable copper, zinc, and manganese in soils of the Canterbury Plains. New Zealand J Agric Res 27, 207-217

Mehlich A (1984) Mehlich 3 soil test extractant: a modification of Mehlich 2 extractant. Commun Soil Sci Plant Anal 15, 1409-1416

Mench M (1993) Notions sur les éléments en traces pour une qualité des sols et des produits végétaux. PURPAN 166, 118-127

Mench M, Juste C, Soldâ P (1992) Effets de I'utilisation de boues urbaines en essai de longue durée : accumulation des métaux par les végétaux supérieurs. Bull Soc Bot Fr 139, Actual bot 1, 141-156

Morel JL, Guckert A (1984) Évolution en plein champ de la solubilité dans le DTPA des métaux introduits par des épandages de boues urbaines. agronomie 4, 377-386

Mulchi CL, Adamu CA, Bell PF, Chaney RL (1992) Residual heavy metal concentrations in sludge amended coastal plain soil. II. Predicting metal concentrations in tobacco from soil test information. Commun Soil Sci Plant Anal 23, 1053-1069

Nirel PMV, Morel FMM (1990) Pitfalls of sequential extractions. Wat Res 24, 1055-1056

Novozamsky I, Lexmond TM, Houba VJG (1993) A single extraction procedure of soil for evaluation of uptake of some heavy metals by plants. Intern $J$ Environ Chem 51, 47-58

O'Connor GA (1988) Use and misuse of the DTPA soil test. J Environ Qual 17, 715-718

Pickering WF (1995) General strategies for speciation. In : Chemical Speciation in the Environment (AM Ure, CM Davidson, eds), Blackie Academic and Professional, Glasgow, 9-32

Prüess A (1992) Vorsorgewerte und Prüfwerte für mobile und mobilisierbare, potentiell ökotoxische Spurenelemente in Böden. Verlag Ulrich E Grauer, Wendligen

Ritchie GSP, Sposito G (1995) Speciation in soils. In : Chemical Speciation in the Environment (AM Ure, CM Davidson, eds), Blackie Academic and Professional, Glasgow, 201-233

Robbe D (1987) Interlaboratory comparison of sequential extraction procedures. In : Heavy Metals in the 
Environment 1 (SE Lindberg, TC Hutchinson, eds), New Orleans, 360-362

Roca J, Pomares F (1991) Prediction of available heavy metals by six chemical extractants in a sewage sludge-amended soil. Commun Soil Sci Plant Anal 22, 2119-2136

Roger B (1986) Comparaison de deux schémas d'extractions sélectives. Phénomènes de réadsorption en cours de minéralisation. Environ Technol Lett 7 , 539-546

Sanders JR, Adams TMcM (1987) The effects of pH and soil type on concentrations of zinc, copper and nickel extracted by calcium chloride from sewage sludge-treated soils. Environ Pollut 43, 219-222

Sanders JR, McGraph SP, Adams TMcM (1987) Zinc, copper and nickel concentrations in soil extracts and crops grown on four soils treated with metalloaded sewage sludges. Environ Pollut 44, 193-210

Sauerbeck DR, Stypereck P (1984) Evaluation of chemical methods for assessing the $\mathrm{Cd}$ and $\mathrm{Zn}$ availability from different soils and sources. In : Chemical Methods for Assessing Bio-Available Metals in Sludges and Soils (R Leschber, RD Davis, P L'Hermite, eds), Elsevier Applied Science Publishers, New York, 49-67

Schneider A (1989) Les méthodes d'analyses de terre par chélation des cations metalliques (EDTA-DTPA). in : Les oligoéléments et le sol ( $P$ Duc, ed), Frontières, Paris, 39-58

Sheppard MI, Thibault DH (1992) Desorption and extraction of selected heavy metals from soils. Soil Sci Soc Am J 56, 2, 415-423

Shuman LM (1988) Effect of organic matter on the distribution of manganese, copper, iron, and zinc in soil fractions. Soil Sci 146, 192-198

Sillanpää M (1982) Micronutrients and the nutrient status of soils: a global study. FAO Soils Bulletin 48 , FAO, Rome

Singh BR, Narwal RP, Jeng AS, Almas Å (1995) Crop uptake and extractibility of cadmium in soils natural- ly high in metals at different $\mathrm{pH}$ levels. Commun Soil Sci Plant Anal 26, 2123-2142

Soltanpour RH, Workman S (1979) Modification of the $\mathrm{NH}_{4} \mathrm{HCO}_{3}$-DTPA soil test to omit carbon black. Commun Soil Sci Plant Anal 10, 1411-1420

Street JJ, Lindsay WL, Sabey BR (1977) Solubility and plant uptake of cadmium in soils amended with cadmium and sewage sludge. J Environ Qual 6, 72-77

Symeonides C, McRae SG (1977) The assessment of plant-available cadmium in soils. J Environ Qual 6, 120-123

Tessier A, Campbell PGC (1991) Comment on 'Pitfalls of sequential extractions' by Nirel PMV, Morel FMM Wat Res 24, 1055-1056 (1990). Wat Res 25, 115117

Tucker MR, Hight PT (1990) A comparison of the results from three soil testing laboratories using the Mehlich-3 extractant on southeastern coastal plain soils. Commun Soil Sci Plant Anal 21, 2197-2208

UNICHIM (Ente Nazionale Italiano di Unificazione) (1991) Method $n^{\circ} 682$ et 683. In : Italian Association for Standardization in the Chemical Industry, UNICHIM, Milan

VSBo (Verordnung über Schadstoffgehalt im Boden) (1986) Swiss Ordinance on Pollutants in Soils $\mathrm{Nr}$ 814.12, Publ Eidg Drucksachen und Materialzentrale, Bern, 1-4

Whalley C, Grant A (1993) Phase selectivity of the BCR sequential extraction procedure. In : Heavy Metals in the Environment 1 (RJ Allan, JO Niagru, eds), Toronto, 289-299

Wu QT (1989) Biodisponibilité du cadmium dans des systèmes sol-plante. Thèse de doctorat de l'Institut national polytechnique de Lorraine

Xiao-Quan S, Bin C (1993) Evaluation of sequential extraction for speciation of trace metals in model soil containing natural minerals and humic acid. Anal Chem 65, 802-807 